\title{
THERAPEUTIC DRUG MONITORING AND LABORATORY MEDICINE
}

\author{
Mike Hallworth and lan Watson. Ed. By Karen Poyser and Roy Sherwood. ACB Venture Publications 2008. 218 \\ pp. ISBN 978 - 0- 902429 - 42 - 0. Price £30.00. Available from the Association of Clinical Biochemists, 1300-132 \\ Tooley St., London SE1 2TU
}

Reviewer: Joseph Lopez, APFCB President, Kuala Lumpur, Malaysia.

Much has changed in therapeutic drug monitoring (TDM) since this book was first published 15 years ago. Many new drugs are now monitored and new or improved measurement technologies have been introduced for their routine measurement. During this time, a new science, pharmacogenomics, has emerged.

This book is an attempt to bring the reader up to date with these changes. It sensibly opens with the theoretical considerations of the discipline and discusses, inter alia, the rationale for TDM pharmacokinetic and pharmacodynamic factors of drug usage. There are also sections on practical considerations for the organisation of an effective TDM service. Each of the five chapters in the book ends with a suggested reading list.

The second chapter is the longest and deals with individual drugs belonging to the following categories: antibiotics, anticancer drugs, anticonvulsants ("the archetypical TDM drugs"), antidepressants, antipsychotics, bronchodilators drugs, cardioactive drugs, drugs of abuse and immunosuppressive drugs. For each drug or sub-category within each of these groups, the clinical use, pharmacokinetics, toxicity and monitoring therapy are discussed. The section on drugs of abuse receives short shrift, perhaps because much is already know about it. (Curiously, amphetamines are spelt "amfetamines", even though this book is published in the UK). The immunosuppressive drugs ciclosporin, mycophenolate, sirolimus and tacrolimus, however, receive the most detailed treatment.

Of interest to those associated with the laboratory would be

\section{Address for Correspondence :}

\section{Mr. Joseph Lopez}

President, APFCB,

Institute for Medical Research, Jalan Pahang 50588

Kualalumpur, Malaysia

E-mail : jblopez@ streamyx.com the chapter on analyses. Early drug assays used methods based on colorimetry or uv absorbance. These methods were often cumbersome lacked sensitivity and specificity. Much of the pioneering work on anticonvulsants was done on gas or liquid chromatography. TDM became

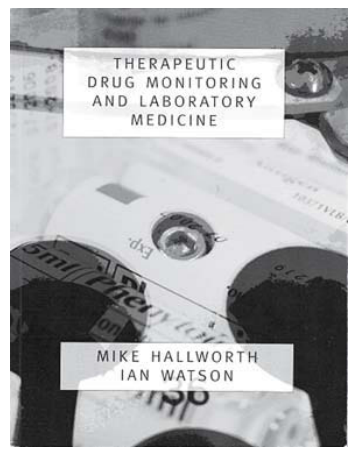
popular in the early 1970 s with the advent of EMIT methods.

Immunoassays were first used in TDM for digitoxin measurement in 1968. However, following the introduction of the "homogeneous" EMIT assays, in the mid-1970s, the foundation was laid for the widespread adoption of immunoassays for TDM. The book discusses some of the a wide variety of immunoassays that are currently available for routine application including those integrated with specific instrument platforms.

While the older, well established drugs were assayed using a variety of immunoassays, the newer TDM drugs such as immunosuppressant needed high performance liquid chromatography-mass spectrometry (HPLC-MS) for measurement. Until fairly recently, HPLC-MS was regarded as an exotic instrument that was confined to the research laboratory. We are informed that an increasing number of laboratories are now turning to LC-tandem-MS because of the superior accuracy of results and convenient routine application. They are particularly useful in monitoring difficult compounds such as immunosuppressants.

Chapter 4 discusses the principles and practice of dosage prediction. The final chapter is on optimising drug therapy. It briefly touches on pharmacogenomics and discusses the development of biomarkers to provide an objective measure of an individual's clinical response to therapeutic intervention.

The book is a timely update of TDM and should serve as an excellent companion to anyone who is involved in it. 